\title{
Image Filtering Algorithm of Subway Tunnel Cracks Based on Regional Difference
}

\author{
Zhengman JIA ${ }^{1}$ and Zhenhai ZHANG \\ School of Automation and Electrical Engineering, Lanzhou Jiaotong University
}

\begin{abstract}
Aiming at the problems of uneven illumination, low contrast and serious noise interference in subway tunnel images, an adaptive median filtering algorithm based on regional differences is proposed to improve noise detection and noise filtering. The algorithm first used the filter window set in advance by the algorithm to detect and determined the noise point by calculating the gray difference in the window. Then it is only filtered by the effective pixels are median-calculated in the template. The result is output as the gray value in the center of the window. Compared with the traditional median, mean and adaptive median filtering algorithms, the proposed new algorithm can effectively filter out noise while reducing the difficulty of subsequent segment recognition.
\end{abstract}

Keywords. Subway tunnel, regional difference, noise detection, effective pixels, adaptive median filter

\section{Introduction}

The construction of subway tunnels in our country has entered a period of rapid development. As time goes by, the subway tunnels will have cracks due to material and temperature during use, which directly affects the stability of the tunnel and the safety of train operation. It must be tested effectively [1]. The detection of tunnel cracks in my country mainly relies on manual marking, which has low efficiency, heavy workload, and is greatly affected by subjective factors, and the accuracy is not high. Crack detection technology based on image processing has gradually become the main development direction due to its high real-time advantages [2].

At present, filter denoising based on image processing had achieved many research results. Chen Jian et al. constructed a directional template for multiple filtering to remove noise and protect edge details [3]. Lu Ziwei et al. detected the location of cracks by extracting the grayscale, entropy and texture feature information of different areas of the image [4]. Zhu Liqiang and others divided noise into noises with different characteristics, then processed them separately to filter out the noise [5]. Zhou Lijun extracted the cracks by dividing the split image cracks and the surrounding grayscale differences [6]. Shuai Murong et al. proposed an improved adaptive median filter to

1 Zhengman JIA, School of Automation and Electrical Engineering, Lanzhou Jiaotong University, China; E-mail: 2801094820@qq.com. 
increase the noise threshold when detecting noise to filter images [7-9]. Si Shaohui et al. proposed a Gaussian filtering denoising algorithm based on irregular regions, which effectively improved the problem of image detail loss [10]. Wang Songlin adopted an improved adaptive weighted median filter algorithm to remove salt and pepper noise [11]. Zhang Xuming proposed an adaptive median-average hybrid filter to separate salt and pepper noise from Gaussian noise, but it is not ideal for image removal with large noise density. This method judges the position of noise points according to the intensity value of pixels, and then performs step by step on the noise pixels within the processing frame of loop iteration [12]. Qu Zhengeng et al. proposed a filtering algorithm that adaptively changes the size of the filter window according to the number of noise points [13].

To this end, the author proposed an adaptive median filtering algorithm based on regional differences to filter out noise. The algorithm worked on the inverted image. Then, the gray difference of the domain is used to determine the noise, and only the effective pixels in the window is operated. It has a good denoising effect and lays a good foundation for crack segmentation and feature extraction. The specific process is shown in Figure 1.

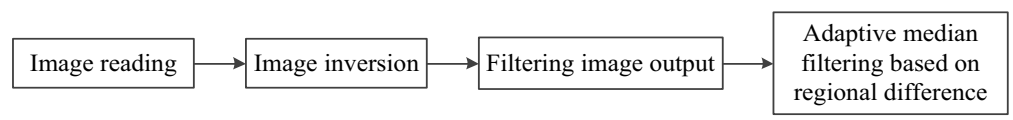

Figure 1. Denoising process of crack image in subway tunnel

\section{Image Inversion}

Image inversion is to invert the gray level of the image, which is suitable for enhancing the white or gray areas in the image. Suppose the gray level of a picture is $[0, \mathrm{~L}-1], \mathrm{s}$ and $\mathrm{r}$ represent the gray value before and after processing, then the inverted image can be obtained by Eq. (1)[14].

$$
s=L-1-r
$$

Due to the uneven illumination and low contrast of the crack images of subway tunnels, this paper uses image inversion to preprocess the crack images. Experiment with histogram equalization, uniform stretching and image inversion, as shown in Figure 2. Taking Gaussian filtering as an example, the original image and the inverted image are filtered separately, as shown in Figure 3. The calculated PSNR (peak signal to noise ratio) and $M S E$ (mean square error) results are compared. The results are shown in Table 1. The definitions of PSNR and MSE are shown in Eqs. (2) and (3).

$$
P S N R=10 \lg \left[\frac{\left(2^{n}-1\right)^{2}}{M S E}\right]
$$




$$
M S E=\frac{1}{n^{2}} \sum_{i=1}^{n} \sum_{j=1}^{n}\left(f_{i, j}^{\prime}-f_{i, j}\right)^{2}
$$

In the formula, $f_{i, j}^{\prime}$ indicates the gray value of the image pixel after processing, $f_{i, j}$ indicates the gray value of the image pixel before processing. $n=8$.

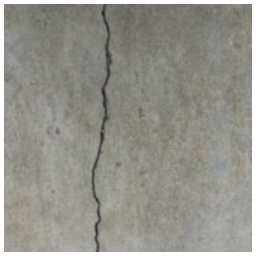

(a) Original image

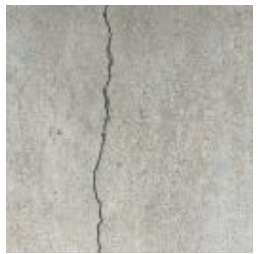

(b)Histogram equalization

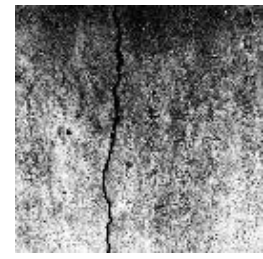

(c) Uniform stretching

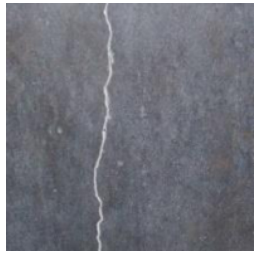

(d) Reverse image

Figure 2. Comparison of different pretreatment methods

It is known from Figure 2 that uniform stretching and histogram equalization stretch the gray value of the image in a global range, which is not conducive to the filtering and segmentation of subsequent images. The image reversal enhances the contrast of the cracks to a certain extent.

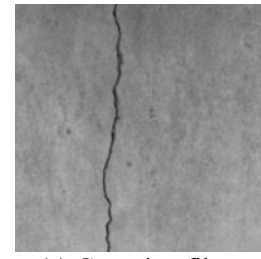

(c) Gaussian filter

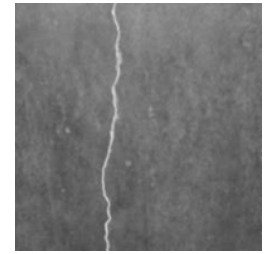

(d) Gaussian filter after inversion

Figure 3. Gaussian filtering and post-inversion filtering

It can be seen from Table 1 that the PSNR of the inverted image after median filtering is higher than that of the original image, and the MSE is smaller than the original image, indicating that the preprocessing effect of this paper is better.

Table 1. Comparison of filtering effects

\begin{tabular}{lll}
\hline & $P S N R$ & $M S E$ \\
\hline Original value filtering & 28.484 & 81.547 \\
Inverse image Gaussian filtering & 29.809 & 54.830 \\
\hline
\end{tabular}




\section{Adaptive Median Filtering Based on Regional Differences}

\subsection{Discrimination of Noise Points}

In an image, the change of image gray value can be calculated by calculating the regional difference between pixel areas. By setting the threshold $\mathrm{T}$, the gray difference between adjacent pixels in the four directions is calculated [15]. Compared with the threshold $\mathrm{T}$ to determine whether the pixel in the current window is a noise point.

The size of the discriminant window is set to $w$ and the set is defined as $G=\left\{G_{k}: k=1, \ldots 4\right\}$, the difference between the discriminant window centered on $(i, j)$ and the pixels in all directions is defined as $G_{k}$. The function expression is shown in Eqs. (4) and (7).

$$
\begin{aligned}
& G_{1}=\left|\frac{1}{w-2} \sum_{z=-\left(\frac{w-3}{2}\right)}^{\left(\frac{w-3}{2}\right)} X_{i+z, j+z}-X_{i-\frac{w-1}{2}, j-\frac{w-1}{2}}\right|+\left|\frac{1}{w-2} \sum_{z=-\left(\frac{w-3}{2}\right)}^{\left(\frac{w-3}{2}\right)} X_{i+z, j+z}-X_{i+\frac{w-1}{2}, j+\frac{w-1}{2}}\right| \\
& G_{2}=\left|\frac{1}{w-2} \sum_{z=-\left(\frac{w-3}{2}\right)}^{\left(\frac{w-3}{2}\right)} X_{i+z, j-z}-X_{i-\frac{w-1}{2}, j+\frac{w-1}{2}}\right|+\left|\frac{1}{w-2} \sum_{z=-\left(\frac{w-3}{2}\right)}^{\left(\frac{w-3}{2}\right)} X_{i+z, j-z}-X_{i+\frac{w-1}{2}, j-\frac{w-1}{2}}\right| \\
& G_{3}=\left|\frac{1}{w-2} \sum_{z=-\left(\frac{w-3}{2}\right)}^{\left(\frac{w-3}{2}\right)} X_{i+z, j}-X_{i-\frac{w-1}{2}, j}\right|+\left|\frac{1}{w-2} \sum_{z=-\left(\frac{w-3}{2}\right)}^{\left(\frac{w-3}{2}\right)} X_{i+z, j}-X_{i+\frac{w-1}{2}, j}\right| \\
& G_{4}=\left|\frac{1}{w-2} \sum_{z=-\left(\frac{w-3}{2}\right)}^{\left(\frac{w-3}{2}\right)} X_{i, j-z}-X_{i, j-\frac{w-1}{2}}\right|+\left|\frac{1}{w-2} \sum_{z=-\left(\frac{w-3}{2}\right)}^{\left(\frac{w-3}{2}\right)} X_{i, j-z}-X_{i, j+\frac{w-1}{2}}\right|
\end{aligned}
$$

Where $G_{1}$ is the difference between positive diagonal pixels, $G_{2}$ is the difference between opposite diagonal lines, $G_{3}$ is the difference between horizontal directions, $G_{4}$ the difference between vertical directions.

If the area difference around the current pixel is relatively large, it means that the point is a noise point. If the area difference around the current pixel is relatively small, the point is an edge point. If the regional difference is less than a certain threshold, the point is disturbed by noise. It is necessary to increase the discrimination window and perform noise discrimination again.

The thresholds $T_{1}, T_{2}$ is set. When the area difference in a certain direction is less than $T_{2}$, the pixel is continuous in that direction. If the area difference in a certain direction is less than $T_{1}$, the pixel is discontinuous. The $T_{2}, T_{1}$ can be adjusted according to the specific image. Specific steps are as follows:

- If all the elements of the set $G$ are greater than $T_{2}$ or less than $T_{1}$, it means that the pixel $X_{i, j}$ is a noise point. 
- If all the elements of the set $G$ are less than $T_{2}$, the pixel $X_{i, j}$ is judged as an edge.

- If the three elements of the set $G$ are less than $T_{2}$ and greater than $T_{1}$, then the pixel is disturbed by the noise. The size of the discrimination window $w$ should be increased with the two-step size to re-discriminate whether the pixel is a noise.

\subsection{Operate on Effective Pixels}

According to the noise discrimination algorithm, the pixels can be divided into noise points and edge points, and then the distance between the effective pixel points and the center point of the template can be used to quickly determine the size of the optimal filter window and operate on the noise points, as shown in Figure 4.

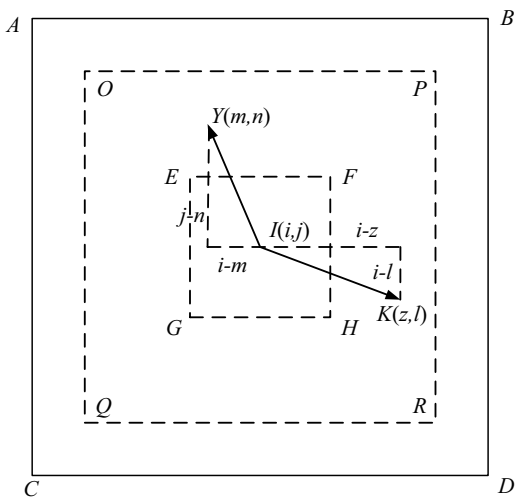

Figure 4. Quickly determine the size of the filter window

As shown in the Figure 4, the size of the window is determined for the distance $r$ between the center point $I$ and the effective pixel point $Y$ coordinate. In order to prevent errors in the window value. The larger the difference between the horizontal and vertical coordinates should be selected as the basis, which can make the number of sorts reduce. As shown in Figure 4, $|j-n|>|i-m|$, the larger one is selected. The $Y$ is divided into $M I N O$, the same is true for point $H$.

First the largest filter window is used to traverse the pixels. Then the best filter window was determined by the distance between the center point of the largest filter and the effective pixels. The effective pixels are sorted in the best filter window to choose the best value. As shown in Figure 5.

\begin{tabular}{|c|c|c|}
\hline 0 & 123 & 0 \\
\hline 0 & 0 & 89 \\
\hline 0 & 100 & 0 \\
\hline
\end{tabular}

(a) Noise window

\begin{tabular}{|c|c|c|}
\hline 0 & 123 & 0 \\
\hline 0 & 0 & 89 \\
\hline 0 & 100 & 0 \\
\hline
\end{tabular}

(b) Traditional filter window

\begin{tabular}{|c|c|c|}
\hline 0 & 123 & 0 \\
\hline 0 & 89 & 89 \\
\hline 0 & 100 & 0 \\
\hline
\end{tabular}

(c) Filter window in this paper

Figure 5. Operate on effective pixels 


\section{Experimental Results and Analysis}

In order to verify the effectiveness of the algorithm in this paper, compared with median filter, mean filter and adaptive median filter, the filter template window is $3 \times 3$, and the subway tunnel image filter is shown in Figure 6 .
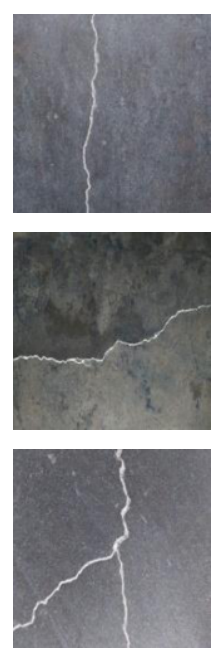

(a) Image inversion
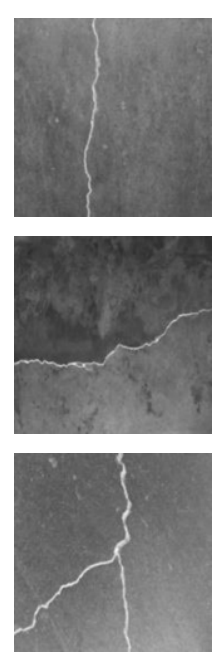

(b) Mean filtering
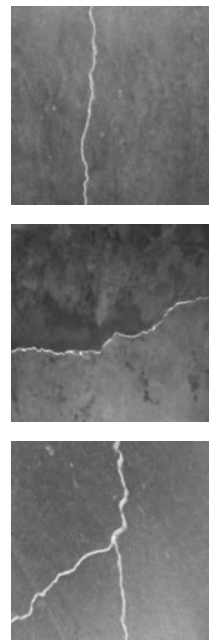

(c) Median filtering
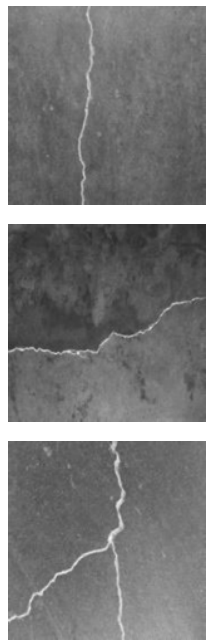

(d) Adaptive median filtering
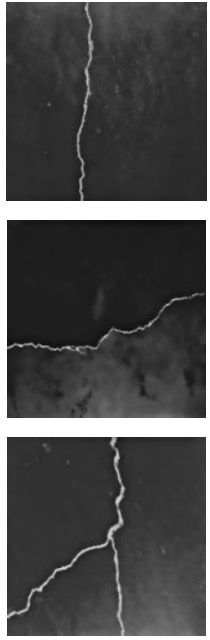

(e) The proposed algorithm

Figure 6. Comparison of simulation results

The filtered image effect can be analyzed from Figure 6. The mean and median filtering will cause the blurring of the crack edge. The difference between the crack and the background is small after the adaptive median filtering. The background part highlights the crack target area after the improved adaptive median filtering, which is conducive to subsequent image segmentation.

In order to verify the effectiveness of the algorithm, the Peak Signal-to-Noise Ratio (PSNR) and running time $(t)$ of the four different filtering algorithms were calculated, and the performance indicators are shown in Table 2.

Table 2. Performance evaluation table of various algorithms

\begin{tabular}{lllll}
\hline & \multicolumn{1}{c}{$\boldsymbol{P S N R}$} & & \multicolumn{1}{l}{ /s } \\
\hline Mean filtering & 28.655 & 27.812 & 28.106 & 0.027 \\
Median filtering & 28.484 & 27.142 & 28.053 & 0.273 \\
Adaptive median & 29.091 & 28.779 & 29.625 & 0.436 \\
Improved filtering & 30.938 & 29.560 & 29.849 & 0.346 \\
\hline
\end{tabular}

It can be seen from Table 2 that the improved filtering algorithm has the highest peak signal-to-noise ratio and the filtering effect is the best. Compared with adaptive median filtering, the running time is also improved. The filtered image is segmented using the same Otsu method, and the segmentation result is shown in Figure 7. 


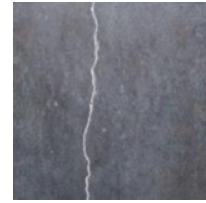

(a)

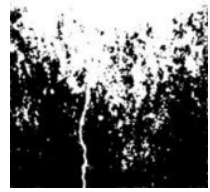

(b)

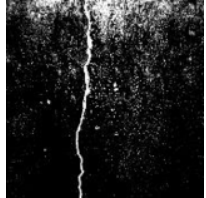

(c)

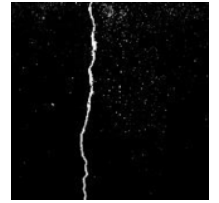

(d)

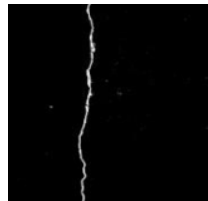

(e)

Figure 7. Comparison of the effects of different filtering algorithms

Figure 7(a) is the original. From Figure 7(b), the image is segmented after mean filtering. Although the mean filtering effect is very good, the background and the target cannot be clearly distinguished after segmentation. Figure 7(c) and Figure 7(d) are segmented images after median filtering and adaptive median filtering. The segmented images still contain a lot of noise. It can be seen from Figure 7(e) that the algorithm not only effectively removes the noise, but also can identify the complete crack area.

\section{Conclusion}

In order to effectively remove the mixed noise in the image, an adaptive median filter based on regional difference is proposed. The advantage of this algorithm is to use the gray difference in the pixel field to distinguish the noise points. And only the effective pixels of the filter window are sorted and calculated to use as a filter output. in the filter window. Its adaptive window filtering improves the efficiency and accuracy of noise detection. Compared with the other four algorithms, the algorithm in this paper not only shows that the image details are clear in subjective vision, but also shows good filtering performance on the peak signal-to-noise ratio and running time index.

\section{Acknowledgment}

This research was supported by the National Natural Science Foundation of China (Grant No.61763025), Natural Science Foundation of Gansu (Grant No.18JR3RA124), China Postdoctoral Science Foundation funded project (Grant No.167306).

\section{References}

[1] Wang YD, Yu ZJ, Bai B, Xu XN, Zhu LQ. Research on crack recognition algorithm of subway tunnel based on image processing. Chinese Journal of Scientific Instrument, 2014, 35(07): 1489-1496.

[2] Zhang ZH, Yin XZ, Ren Q. Filtering algorithm of subway tunnel crack image based on adaptive mean. Journal of Chongqing Jiaotong University (Natural Science Edition), 2019, 38(06): 1-5.

[3] Chen J, Zheng SH, Yu L, Pan L. An improved multi-threshold adaptive median filtering algorithm based on direction. Journal of Electronic Measurement and Instrument, 2013, 27(02): 156-161.

[4] Lu ZW, Wu CD, Chen DY, Shang SB. Pavement crack detection algorithm based on multi-scale analysis of subregions. Journal of Northeastern University (Natural Science Edition), 2014, 35(05): $622-625$.

[5] Zhu LQ, Bai B, Wang YD, Yu ZJ, Guo BQ. Metro tunnel crack recognition algorithm based on feature analysis. Journal of the China Railway Society, 2015, 37(05): 64-70. 
[6] Zhou LJ. Low-contrast crack extraction method for tunnels based on image enhancement and watershed segmentation. Computer Science, 2018, 45(S1): 259-261

[7] Shuai MR, Liao XY, Cheng H, et al. An adaptive median filtering algorithm with improved mean value. Bulletin of Surveying and Mapping, 2019,000(003):53-56,90.

[8] Lan X, Liu XX, Shen HF, et al. An iterative median filtering algorithm to eliminate high-density salt and pepper noise. Journal of Wuhan University Information Science Edition, 2017, 42(12): 1731-1737.

[9] Zhang PX. An adaptive weighted mean filtering algorithm based on salt and pepper noise. Shanghai: East China Normal University 2015.

[10] Si SH, Hu FY, Gu YJ, et al. A Gaussian filter denoising algorithm based on irregular regions. Computer Science, 2014, 41(11):313-316.

[11] Wang SL, Jiang Z. Improved adaptive weighted median filter algorithm. Sensors and Microsystems, 2016, 35(11): 128-131.

[12] Zhang XM, Xu BS, Dong SY, et al. Adaptive median-weighted mean hybrid filter. Optical Technology, 2004, 30(6):652-655.

[13] Qu ZG, Niu SQ. Research on an improved adaptive weighted median filter algorithm. Computer Technology and Development, 2018, 28(12):92-96.

[14] Ruan QQ. Digital Image Processing. 3rd Edition. Electronics Industry Press, 2013.

[15] Jiang QL, Liu S, Chen X. Real-time face localization based on difference area. Journal of Liaoning Technical University (Natural Science Edition), 2011, 030(003):434-437. 